\title{
ANALYTIC SENSING: NONITERATIVE RETRIEVAL OF POINT SOURCES FROM BOUNDARY MEASUREMENTS*
}

\author{
D. KANDASWAMY ${ }^{\dagger}$, T. BLU ${ }^{\ddagger}$, AND D. VAN DE VILLE ${ }^{\dagger}$
}

\begin{abstract}
We consider the problem of locating point sources in the planar domain from overdetermined boundary measurements of solutions of Poisson's equation. In this paper, we propose a novel technique, termed "analytic sensing," which combines the application of Green's theorem to functions with vanishing Laplacian - known as the "reciprocity gap" principle - with the careful selection of analytic functions that "sense" the manifestation of the sources in order to determine their positions and intensities. Using this formalism we express the problem at hand as a generalized sampling problem, where the signal to be reconstructed is the source distribution. To determine the positions of the sources, which is a nonlinear problem, we extend the annihilating-filter method, which reduces the problem to solving a linear system of equations for a polynomial whose roots are the positions of the point sources. Once these positions are found, resolving the according intensities boils down to solving a linear system of equations. We demonstrate the performance of our technique in the presence of noise by comparing the achieved accuracy with the theoretical lower bound provided by Cramér-Rao theory.
\end{abstract}

Key words. Cauchy boundary problem, analytic functions, annihilating filter, nonlinear estimation polynomial

AMS subject classifications. 15A29, 31A25, 31A30

DOI. $10.1137 / 080712829$

1. Introduction. Source imaging from boundary Cauchy data satisfying the Laplace equation is a classical inverse problem that is of high interest to many fields in engineering. Unfortunately, the problem is ill-posed and additional assumptions about the source configuration are needed to make the solution unique. Typically, one can restrict the class of source distributions by imposing smoothness properties (e.g., Tikhonov regularization [19]) or by imposing a parametric source model.

It is when the source configurations are expected to be "sparse" that parametric solutions may prove very useful. This situation is particularly attractive in the electromagnetic setting for applications such as electroencephalography (EEG); e.g., the localization of some type of epileptic foci can be reasonably modeled by pointwise (dipolar) sources [15]. Almost all known techniques rely on the forward model; i.e., computing the boundary data for a given source configuration and iteratively fitting its parameters by least-squares optimization [16]. The corresponding cost function has many local minima and makes the solution very sensitive to the initial guess. Therefore, successful recovery of the parametric sources is often limited to single-dipole models.

Despite the practical difficulties in identifying parametric source models, the mathematical uniqueness of the solution has been proven [7], and stability results are available for the case of dipolar and point sources in 2D [5, 16] and 3D [20]. Instead

\footnotetext{
* Received by the editors January 9, 2008; accepted for publication (in revised form) April 20, 2009; published electronically July 31, 2009. This work partially supported by the Swiss National Science Foundation under grant 200021-119812 and in part by the Center for Biomedical Imaging (CIBM) of the Geneva-Lausanne Universities and the EPFL as well as the foundations Leenaards and Louis-Jeantet.

http://www.siam.org/journals/sisc/31-4/71282.html

†École Polytechnique Fédérale de Lausanne (EPFL), Lausanne, Switzerland (Djano. Kandaswamy@epfl.ch, Dimitri.VanDeVille@epfl.ch).

$\ddagger$ Chinese University of Hong Kong, Shatin, Hong Kong (thierny.blu@m4x.org).
}

3179 
of relying on the computation of the forward model, several other approaches have been proposed based on the properties of functional analysis in the complex plane. These methods are planar by their nature, but they can be extended to 3D; e.g., for the axisymmetric setting or by combining the results of multiple cross-sections. In particular, Baratchart et al. [2, 3] have chosen to approximate the known boundary data for monopolar and dipolar sources by a meromorphic function which, under the source model hypothesis, has poles that can be related to the sources' positions.

Another interesting approach uses a ubiquitous tool in electromagnetism, known as the "reciprocity gap" concept by Andrieux and Ben Abda [1] who applied it to the inverse planar crack problem. This tool is essentially an application of Green's theorem, which provides a way to transform a scalar product between the source distribution and a function with vanishing Laplacian into a boundary integral. El Badia et al. have shown that the application of this tool to powers of $x+i y$ leads to an algorithm that solves the localization problem $[6,7]$. Unfortunately, the practical efficiency of their approach to multipole sources retrieval is severely hindered by the numerical instability of higher degree monomials.

In this work, we develop a new algorithmic framework that allows us to identify parametric source models. We term our method "analytic sensing" because it relies heavily on a careful choice of analytic test functions - sensors [10, 11, 12]. Our contributions are twofold:

- We apply the reciprocity gap concept to test functions that are not analytic outside the domain of interest; i.e., unlike the polynomials considered in [7], these "analytic sensors" have a spatial localization that allows one to "sense" the influence of nearby pointwise sources.

- We solve the associated nonlinear problem for the source localization by adapting the generalized annihilation technique developed for sampling signals that have a finite rate of innovation $[21,4]$.

The approach brings together several attractive features: (1) the nonlinear (the localization) and linear (determining the intensities) estimation steps are decoupled; (2) the nonlinear estimation is direct (noniterative) and fast; (3) the solution to the forward problem is not needed; (4) the method can be spatially selective to only incorporate the influence of sources in a desired region-of-interest. We also investigate the accuracy and stability of the method under various noise conditions by evaluating the Cramér-Rao bounds.

2. Problem setting. Let us consider a closed domain $\Omega$ with boundary $\partial \Omega$ in 2D. Moreover, consider a source distribution $\rho$ in $\Omega$. The source distribution induces an electrical potential $V$ such that [9]:

$$
\begin{aligned}
-\operatorname{div}(\sigma \nabla V) & =\rho, \quad \text { within } \Omega, \\
\nabla V \cdot \mathbf{e}_{\Omega} & =0, \quad \text { on } \partial \Omega,
\end{aligned}
$$

where $\sigma$ expresses the conductivity profile of the environment $\Omega$, and where $\mathbf{e}_{\Omega}$ is the outward normal to $\partial \Omega$ as illustrated in Figure 1. When $\sigma$ is constant, (1) is equivalent to Laplace's equation.

Many physical problems can be modeled using (1), and in particular electrostatic problems. Here, the Von Neumann condition on the boundary (2) specifies that no current can flow out of $\Omega$ [9]. Usually, in addition to (1) and (2), one has access to direct measurements of $V$ on $\partial \Omega$. This means that the system is overdetermined since, in theory, given $\rho$ and $V \mid \partial \Omega$ (1) has a unique solution $V$ in $\Omega$, known as the Dirichlet problem [17]. 


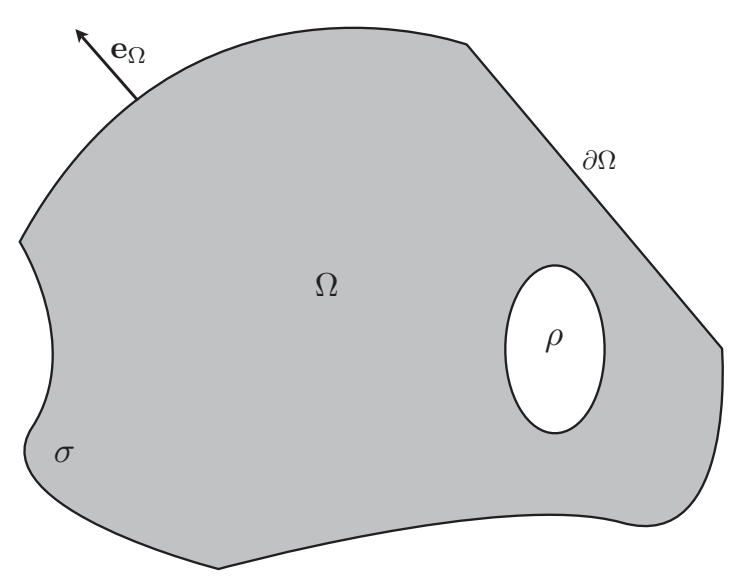

FIG. 1. A homogeneous $2 D$ conductor $\Omega$ with boundary $\partial \Omega$, source distribution $\rho$, constant conductivity $\sigma$, and outward surface normal $\mathbf{e}_{\Omega}$.

However, the problem at hand does not assume the source distribution $\rho$ to be known. On the contrary, the goal is to determine the sources responsible for the electric potential $\left.V\right|_{\partial \Omega}$ measured on the boundary $\partial \Omega$. Thus, the problem here is the following:

$\mathcal{P}$ : Knowing $\left.V\right|_{\partial \Omega}$, find $\rho$ enclosed by $\partial \Omega$, such that (1) and (2) are satisfied.

This problem is not well-posed as there are many possible source fields that solve $\mathcal{P}$ with the same measurements [2]. This can be seen by an example when $\Omega$ is a disk of radius 1: consider a function $V$ which satisfies ${ }^{1} V(\mathbf{x})=0$ and $\mathbf{x}^{\mathrm{T}} \nabla V(\mathbf{x})=0$ on the boundary $\|\mathbf{x}\|=1$. Given our boundary measurements, we may reasonably infer that $\rho=0$ is the solution to $\mathcal{P}$, but $\rho=\operatorname{div}(\sigma \nabla V)$ is a solution as well. It will be thus necessary to severely restrict the class of source fields to be retrieved.

3. Analytic sensing. We first develop our new framework in 2D. It uses "test" functions $\psi(\mathbf{x})$ such that

$$
\operatorname{div}(\sigma \nabla \psi)=0, \quad \text { within } \Omega,
$$

to determine the manifestation of the source distribution in $\Omega$. When $\sigma$ is constant in $\Omega$, this reduces to the functions whose Laplacian is zero in $\Omega$, a large subset of which are $\Omega$-analytic functions. The fundamental observation is that if $V$ is known on the boundary $\partial \Omega$, then we can exactly calculate the scalar products $\langle\psi, \rho\rangle=$ $\int_{\Omega} \psi(\mathbf{x}) \rho(\mathbf{x}) \mathrm{d}^{2} \mathbf{x}$ for such test functions, as shown by the following theorem.

THEOREM 1. Let $V$ be the steady-state potential induced by some source distribution, $\rho$, according to (1) and (2). Moreover, suppose that we know $V(\mathbf{x})$ on the boundary $\mathbf{x} \in \partial \Omega$. Then, if we choose $\psi$ according to (3), the scalar product $\langle\psi, \rho\rangle$ can be expressed as a line integral according to

$$
\langle\psi, \rho\rangle=-\int_{\partial \Omega} \sigma V \nabla \psi \cdot \mathbf{e}_{\Omega} \mathrm{d} s .
$$

The scalar products $\langle\psi, \rho\rangle$ can be seen as "generalized" samples of the unknown distribution $\rho$.

\footnotetext{
${ }^{1}$ For instance, we could choose $V(\mathbf{x})=\left(\|\mathbf{x}\|^{2}-1\right)^{2}$.
} 
Proof. We have the following identity:

$$
\psi \operatorname{div}(\sigma \nabla V)-V \operatorname{div}(\sigma \nabla \psi)=\operatorname{div}(\sigma \psi \nabla V-\sigma V \nabla \psi) .
$$

If we choose $\psi$ to be a test function that satisfies (3), then, by applying (1), this identity becomes

$$
\psi \rho=\operatorname{div}(\sigma \psi \nabla V-\sigma V \nabla \psi) .
$$

Integrating both sides over $\Omega$ and applying Green's divergence theorem yields

$$
\langle\psi, \rho\rangle=\int_{\partial \Omega}(\sigma \psi \nabla V-\sigma V \nabla \psi) \cdot \mathbf{e}_{\Omega} \mathrm{d} s .
$$

The Von Neumann boundary condition (2) further simplifies the left-hand side to (4), which proves the theorem.

It is potentially beneficial to describe $\rho$ through a large collection of measurements $\langle\psi, \rho\rangle$. For instance, if we could compute these scalar products for any $\psi \in \mathcal{D}(\Omega)$, the set of infinitely differentiable functions in $\Omega$, then the generalized samples $\langle\psi, \rho\rangle$ would represent $\rho$ completely and uniquely in the sense of distributions.

Unfortunately, the test functions are here restricted to those that $\operatorname{satisfy} \operatorname{div}(\sigma$ $\nabla \psi)=0$. These functions form a much more constrained class that is unable to characterize the full generality of source fields; an issue that is a direct consequence of the ill-posedness of problem $\mathcal{P}$. Note that, a priori, we can choose any test function that satisfies (3). These test functions form the link between the boundary measurements on $\partial \Omega$ and the generalized samples $\langle\mu, \rho\rangle$. In practice, an interpolation model is needed to evaluate (4) for a limited number of boundary measurements.

3.1. Homogeneous medium. In this paper, we consider in detail only the case where $\sigma$ is constant in $\Omega$. Nevertheless, it is possible to accommodate for varying $\sigma$ as explained briefly in 3.4 .

Thanks to the homogeneity hypothesis, the set of test functions satisfying (3) is made of $\Omega$-harmonic functions, a large subset of which are functions that are analytic in $\Omega$, e.g., polynomials in $z=x+i y$, which were used [7]. We will opt for rational functions of $z$ that do not have any poles in $\Omega$ and coin the term "analytic sensors."

COROLlary 1. Let $\psi$ be an analytic sensor. If we use the analytic formalism, then we can compute the associated generalized sample, $\mu=\langle\psi, \rho\rangle$, as follows:

$$
\mu=i \sigma \int_{\partial \Omega} V(x, y) \psi^{\prime}(z) \mathrm{d} z
$$

Proof. If $\psi$ is an analytic function, i.e., a differentiable function of $z=x+i y$, then $\nabla \psi=\psi^{\prime}(z)[1, i]^{\mathrm{T}}$. Moreover, $\mathbf{e}_{\Omega} \mathrm{d} s=[\mathrm{d} y,-\mathrm{d} x]^{\mathrm{T}}$ which implies that $\nabla \psi \cdot \mathbf{e}_{\Omega} \mathrm{d} s=$ $-i \psi^{\prime}(z) \mathrm{d} z$. By application of Theorem 1 this proves (8).

As said before, we need to further restrain $\rho$ so that $\mathcal{P}$ becomes well-posed. Here, we assume that $\rho$ consists of pointwise sources. Hence, we parametrize $\rho$ such that:

$$
\rho(\mathbf{x})=\sum_{m=1}^{M} c_{m} \delta\left(\mathbf{x}-\mathbf{x}_{m}\right)
$$

with $c_{m} \in \mathbb{R}, M \in \mathbb{N}$, and $\mathbf{x}_{m} \in \mathbb{R}^{2}$. Furthermore, if we choose $\psi(z)=1$ and use Theorem 1, then it is clear that the following should hold:

$$
\sum_{m=1}^{M} c_{m}=0 .
$$

Copyright @ by SIAM. Unauthorized reproduction of this article is prohibited. 


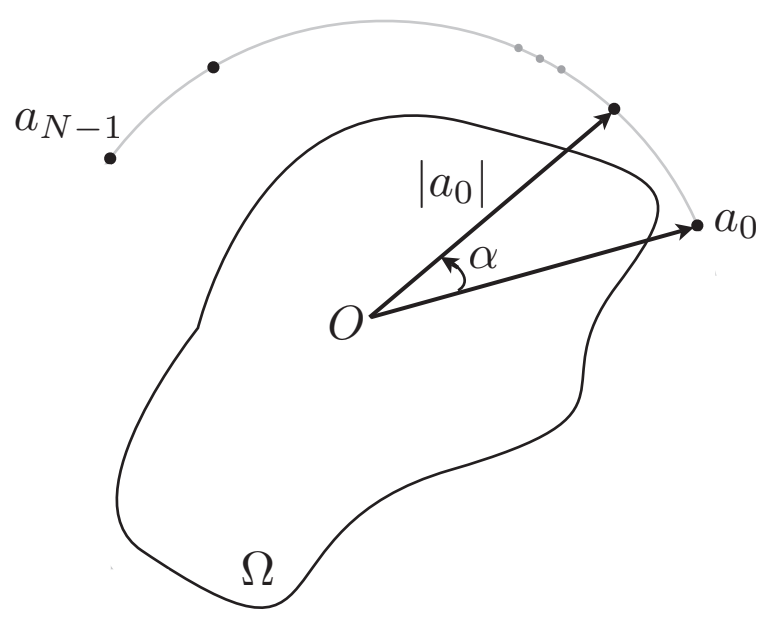

FIG. 2. The poles, $a_{0} \cdots a_{N-1}$, and their placement outside of $\Omega$. For each pole there is a corresponding analytic sensor $\psi_{a_{n}}(z)=\frac{1}{z-a_{n}}$.

This parametrization is typical for a "finite rate of innovation" setting [21]. We will see that this setting ensures that $\mathcal{P}$ is well-posed - or at least, that if it has a solution, this solution is unique [2]. Thus, the purpose of solving the inverse problem at hand shifts towards the retrieval of the positions and the intensities of the pointwise sources.

3.2. Choice of the test functions. Now that the problem is well-formulated and well-posed, we need to choose the sensors in a way that the corresponding generalized samples allow us to determine algorithmically the positions and the intensities of the pointwise sources. For that purpose, as it will become clear in the next section, we choose the following family of test functions:

$$
\psi_{a_{n}}(z)=\frac{1}{z-a_{n}}, \quad a_{n} \notin \Omega .
$$

More specifically, we will restrict further our choice of $a_{n}$ to the form $a_{n}=a_{0} e^{i n \alpha}$, where $n \in \llbracket 0, N-1 \rrbracket^{2}$ for some $N>2 M$ and $\left.\alpha \in\right] 0,2 \pi\left[\right.$. The radius $\left|a_{0}\right|$ is chosen such that $a_{n} \notin \Omega$. This setup is depicted in Figure 2. Note that the angle $\alpha$ is completely arbitrary; in particular, we do not need $N \alpha$ to be equal to $2 \pi$. Actually, $N \alpha$ could even be close to 0 , meaning that the poles of the analytic sensor would all be located in the neighborhood of $a_{0}$.

Another very interesting characteristic of these analytic sensors is that they are "localized," and this all the more as their poles are closer to $\partial \Omega$. This means that it is conceivable to compute a good approximation of the integral in (8) only with values of the potential that are close to the pole of the sensor.

3.3. A note on missing data. In practice, $V$ is not continuously known on the boundary $\partial \Omega$; i.e., we measure $V\left(\mathbf{x}_{n}\right), n \in \llbracket 0, \cdots, N-1 \rrbracket$, where $\mathbf{x}_{n} \in \partial \Omega$. Hence, we need an interpolation/approximation method to reconstruct the continuous-domain representation of $\left.V\right|_{\partial \Omega}$. In order to give an insight of what actually happens, we consider the (very) simplified case where $\partial \Omega$ is a circle with radius 1 and where $V$ is measured at the $N$ uniform angles $\theta_{n}=2 n \pi / N$ for $n=\llbracket 0, N-1 \rrbracket$. With limited

\footnotetext{
${ }^{2} \llbracket a, b \rrbracket$ denotes the set of integers $\{a, a+1, \cdots, b\}$.
} 
ambiguity, we denote by $V(\theta)$ the measure of the electric potential at angle $\theta$ and by $\tilde{V}(\theta)$ its interpolated version. More specifically, we assume $N=2 K+1$ to be odd and develop $V$ and $\tilde{V}$ in Fourier series:

$$
V(\theta)=\sum_{m \in \mathbb{Z}} c_{m} e^{i m \theta}, \quad \text { and } \quad \tilde{V}(\theta)=\sum_{m=-K}^{K} \tilde{c}_{m} e^{i m \theta} .
$$

The coefficients $\tilde{c}_{m}$ are obtained by solving a linear system of equations that expresses the constraints of the measurements $\tilde{V}\left(\theta_{n}\right)=V\left(\theta_{n}\right), n=\llbracket 0, N-1 \rrbracket$ :

$$
V\left(\theta_{n}\right)=\sum_{m=-K}^{K} \tilde{c}_{m} e^{i m \theta_{n}}
$$

Thanks to our specific choices, these coefficients can be expressed directly using a discrete Fourier transform (DFT) of the measurements

$$
\tilde{c}_{m}=\frac{1}{N} \sum_{n=-K}^{K} V\left(\theta_{n}\right) e^{-i m \theta_{n}}=\sum_{n \in \mathbb{Z}} c_{m+N n},
$$

by replacing $V\left(\theta_{n}\right)$ with its Fourier expansion (12). The last identity implies that

$$
\sum_{m=-K}^{K}\left|\tilde{c}_{m}-c_{m}\right| \leq \sum_{|m|>K}\left|c_{m}\right|
$$

and in particular $|\tilde{V}(\theta)-V(\theta)| \leq 2 \sum_{|m|>K}\left|c_{m}\right|$; not so surprisingly, the interpolation error is bounded by (twice) the $\ell^{1}$ norm of the "truncated" Fourier coefficients.

We now consider the generalized measures $\mu_{n}=\left\langle\psi_{a_{n}}, \rho\right\rangle$ with $N$ analytic sensors located at $a_{n}=a_{0} e^{i \theta_{n}}$. Using the Fourier representation (12) of $V(\theta)$ in (8), we have that

$$
\begin{aligned}
\mu_{n} & =i \sigma \int_{\partial \Omega} V(\theta) \psi_{a_{n}}^{\prime}(z) \mathrm{d} z \\
& =i \sigma \sum_{m \in \mathbb{Z}} c_{m} \int_{\Omega} \psi_{a_{n}}^{\prime}(z) e^{i m \theta} \mathrm{d} z \\
& =i \sigma \sum_{m \in \mathbb{Z}} c_{-m} \int_{\Omega} \frac{\psi_{a_{n}}^{\prime}(z)}{z^{m}} \mathrm{~d} z
\end{aligned}
$$

Knowing that $\psi_{a_{n}}$ is analytic in $\Omega$ and using Cauchy's theorem we can deduce the following:

$$
\begin{aligned}
\mu_{n} & =-2 \pi \sigma \sum_{m=1}^{\infty} c_{-m} \frac{\psi_{a_{n}}^{(m)}(0)}{(m-1) !} \\
& =2 \pi \sigma \sum_{m=1}^{\infty} \frac{m c_{-m}}{a_{n}^{m+1}}
\end{aligned}
$$

and finally

$$
e^{i \theta_{n}} \mu_{n}=\sum_{m=-\infty}^{-1}-2 \pi \sigma \frac{m c_{m}}{a_{0}^{-m+1}} e^{i m \theta_{n}}
$$

Copyright $@$ by SIAM. Unauthorized reproduction of this article is prohibited. 


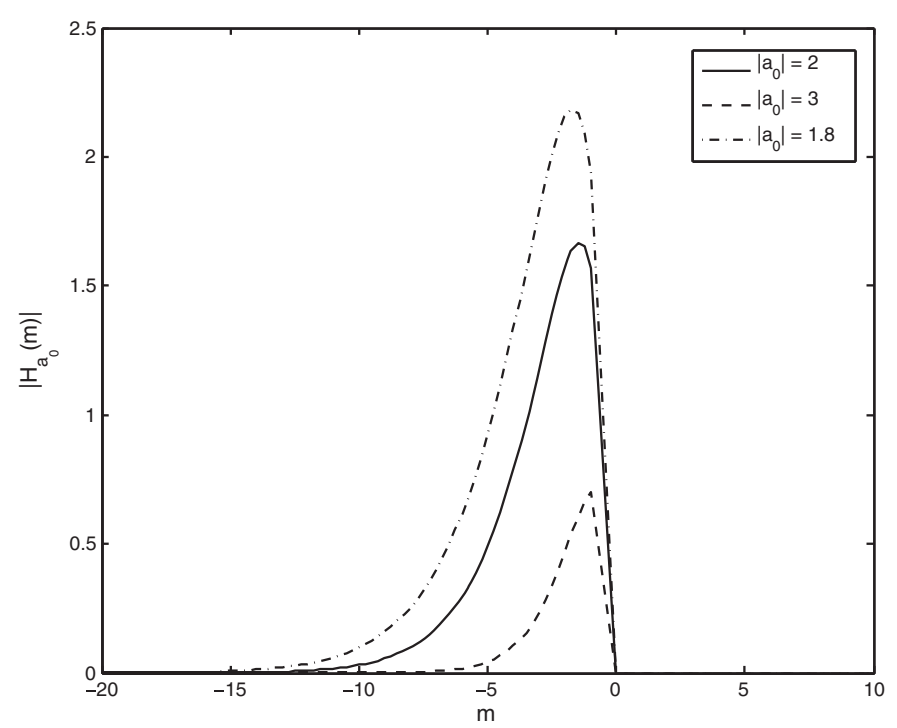

FIG. 3. Magnitude of the frequency response of $H_{a_{0}}(m)$ that is the equivalent filter that links the boundary measures to the generalized measures. Different magnitudes of the pole $a_{0}$ are shown.

Similarly, if we replace $V(\theta)$ by its interpolation $\tilde{V}(\theta)$ in (8) and denote by $\tilde{\mu}_{n}$ the approximated generalized measure, we get

$$
e^{i \theta_{n}} \tilde{\mu}_{n}=\sum_{m=-K}^{-1}-2 \pi \sigma \frac{m \tilde{c}_{m}}{a_{0}^{-m+1}} e^{i m \theta_{n}} .
$$

First, we can bound the error between the actual, computable, generalized measures $\tilde{\mu}_{n}$ and the inaccessible ones $\mu_{n}$ :

$$
\left|\tilde{\mu}_{n}-\mu_{n}\right| \leq \max _{m \geq 1} \frac{2 \pi \sigma m}{\left|a_{0}\right|^{m+1}} \underbrace{\left(\sum_{m=-K}^{-1}\left|\tilde{c}_{m}-c_{m}\right|+\sum_{m<-K}\left|c_{m}\right|\right)}_{\leq 2 \sum_{|m|>K}\left|c_{m}\right|} .
$$

The computation error on the generalized measures is thus directly controlled by the $\ell^{1}$ norm of the out-of-band Fourier coefficients, which is typically small when the function $V(\theta)$ is smooth.

Second, by bringing together (19) and (13), we conclude that the discrete sequence $e^{i \theta_{n}} \tilde{\mu}_{n}$ is a filtered version of $V\left(\theta_{n}\right)$, because the DFT coefficients of each sequence are equal, up to a multiplication by

$$
H_{a_{0}}(m)= \begin{cases}-2 \pi \sigma m a_{0}^{-m-1} & \text { for }-K \leq m<0 \\ 0 & \text { for } m \geq 0 \text { or } m<-K .\end{cases}
$$

The magnitude response $\left|H_{a_{n}}(m)\right|$ is shown in Figure 3 for different values of $\left|a_{0}\right|$. It is maximum for $m \approx-1 / \ln \left|a_{0}\right|$. The filtering appears as a combination of a (flipped) Hilbert transform and band-pass filtering. The bandwidth of the filter increases as the pole's position approaches the boundary. This behavior indicates that $\left|a_{0}\right|$ should be chosen with respect to the noise characteristics of the measurements. 
3.4. A note on nonhomogeneous medium. In the case of a nonhomogeneous medium, we only need to assume that $\sigma$ is constant within the potential support $\Omega_{0} \subset \Omega$ of $\rho$. In that case, we may still choose $\left.\psi\right|_{\Omega_{0}}$ to be of the form $(z-a)^{-1}$. Then, $\psi$ can be propagated into $\Omega \backslash \Omega_{0}$ in such a way as to satisfy $\operatorname{div}(\sigma \nabla \psi)=0$ using numerical techniques such as finite element methods [13] or boundary element methods (for domains with piecewise constant $\sigma$ ) [14]. The propagation of the test functions up to the boundary $\partial \Omega$ implicitly encodes the information of the forward model in more complex configurations. Consequently, the generalized samples also take into account the presence of the nonhomogeneous medium. The localization method as presented in the following section, however, remains identical. Within the context of EEG, one well-known head model is the multilayer sphere [18]. The conductivity is homogeneous in each spherical layer and the dipolar sources are assumed to be in the "gray matter" compartment.

4. A noniterative solution to the localization problem. From now on we shall identify $\mathbf{x}_{m}$ with the complex plane, hence $\mathbf{x}_{m} \Leftrightarrow z_{m}=x_{m}+i y_{m}$. Now, we define the polynomial, $R(X)$, whose roots are the positions of the pointwise sources:

$$
R(X)=\prod_{m=1}^{M}\left(X-z_{m}\right)=\sum_{k=0}^{M} r_{k} X^{k} .
$$

Using (11) and the fact that $a_{n}=a_{0} e^{i n \alpha}$, the following relationship exists between $R$ and the generalized samples $\mu_{n}=\left\langle\psi_{a_{n}}, \rho\right\rangle$ :

$$
\mu_{n}=\sum_{m=1}^{M} c_{m} \psi_{a_{n}}\left(z_{m}\right)=\frac{\sum_{m=0}^{M-1} c_{m}^{\prime} e^{i m n \alpha}}{R\left(a_{n}\right)},
$$

where $c_{m}^{\prime}$ are complex-valued coefficients that do not depend on $n$ or $\alpha$. We now see that the generalized samples satisfy an "annihilating" equation.

LEMMA 1. Consider the finite impulse response digital filter, $h=\left\{h_{k}\right\}_{k \in \mathbb{Z}}$, which has its zeros at $e^{i k \alpha}$ for $k=0,1, \ldots, M-1$. It is characterized by the transfer function

$$
H(z)=\sum_{k \in \mathbb{Z}} h_{k} z^{-k}=\prod_{k=0}^{M-1}\left(1-e^{i k \alpha} z^{-1}\right) .
$$

Then, the filter $h$ annihilates the sequence $u=\left\{u_{n}\right\}_{n=0, \ldots, N-1}$ whose coefficients are defined by $u_{n}=R\left(a_{n}\right) \mu_{n}$; i.e.,

$$
h * u=0, \quad \text { for all } n \in \llbracket M, N-1 \rrbracket .
$$

Proof. Given an integer $m$ in $\{0, M-1\}$, consider the discrete convolution of the sequence $\left\{e^{i m n \alpha}\right\}_{n \in\{0, N-1\}}$ with $h$. For an output index $n \in \llbracket M, N-1 \rrbracket$, its $n$th element is given by the summation:

$$
\sum_{k=0}^{M} h_{k} e^{i m(n-k) \alpha}=e^{i m n \alpha} H\left(e^{i m \alpha}\right)=0 .
$$

On the other hand, by (23) we have $u_{n}=\sum_{m=0}^{M-1} c_{m}^{\prime} e^{i m n \alpha}$ for $n \in \llbracket 0, N-1 \rrbracket$. We can thus write, using the linearity of the discrete convolution, that

$$
\sum_{k=0}^{M} h_{k} u_{n-k}=0
$$

for any output index $n \in \llbracket M, N-1 \rrbracket$, which proves our claim. 
Theorem 2. The coefficients $r_{k}$ of the polynomial $R(X)$ defined by (22) satisfy the following linear system of equations:

$$
\begin{gathered}
\sum_{k=0}^{M} A_{n, k} r_{k}=0, \quad \text { for } n \in \llbracket M, N-1 \rrbracket, \\
\text { where } A_{n, k}=\sum_{n^{\prime}=0}^{N-1} h_{n-n^{\prime}} a_{n^{\prime}}^{k} \mu_{n^{\prime}} .
\end{gathered}
$$

In matrix form, this system can be expressed as $\mathbf{A R}=\mathbf{0}$, if we define the matrix $\mathbf{A}$ by $[\mathbf{A}]_{n, k}=A_{n, k}$ for $n \in \llbracket M, N-1 \rrbracket$ and $k \in \llbracket 0, M \rrbracket$, and the vector $\mathrm{R}=\left[r_{0}, r_{1}, \ldots, r_{M}\right]^{\mathrm{T}}$. Note that we have $r_{M}=1$.

Proof. By combining Lemma 1 and (22), we deduce the following:

$$
\begin{aligned}
0=\{h * u\}_{n} & =\sum_{n^{\prime}=0}^{N-1} h_{n-n^{\prime}} R\left(a_{n^{\prime}}\right) \mu_{n^{\prime}} \\
& =\sum_{n^{\prime}=0}^{N-1} h_{n-n^{\prime}} \sum_{k=0}^{M} r_{k} a_{n^{\prime}}^{k} \mu_{n^{\prime}} \\
& =\sum_{k=0}^{M} r_{k} \underbrace{\sum_{n^{\prime}=0}^{N-1} h_{n-n^{\prime}} a_{n^{\prime}}^{k} \mu_{n^{\prime}}}_{A_{n, k}}
\end{aligned}
$$

for all $n \in \llbracket M, N-1 \rrbracket$, which proves the theorem.

Thus, combining Corollary 1 with Theorem 2 yields a noniterative algorithm for localizing the pointwise sources given boundary measurements. That is, we first obtain the generalized measurements $\mu_{n}=\left\langle\psi_{a_{n}}, \rho\right\rangle$ from the observed boundary measurements, $\left.V\right|_{\partial \Omega}$; then, by computing the polynomial, $R(X)$, according to (24) we are able to find the positions $z_{m}$ by taking its roots. Once the positions of the pointwise sources are known, we need to determine their intensities, $c_{m}$. The generalized samples $\mu_{n}$ depend linearly on the intensities $c_{m}$ as is clear from (23). Hence, determining the intensities boils down to solving the following linear system of equations:

$$
\sum_{m=1}^{M} \frac{c_{m}}{z_{m}-a_{n}}=\mu_{n}, \quad n \in \llbracket 0, N-1 \rrbracket .
$$

Figure 4 schematizes the complete retrieval algorithm.

The linear system in (24) with the additional constraint $r_{M}=1$ make up $N-M$ equations in total. On the other hand, there are $M$ unknown polynomial coefficients $r_{k}, k \in \llbracket 0, M-1 \rrbracket$. Consequently, we do not need more than $N=2 M$ generalized measures $\mu_{n}$ to retrieve the coefficients $r_{k}$. Note that when considering the complete problem, the amplitudes $c_{m}$ and source positions $z_{m}$ make precisely $2 M$ (complex) unknowns for $2 M$ (complex) nonlinear equations (23). For the noiseless case and for $M$ distinct source positions, it may be argued that $\operatorname{rank}(\mathbf{A})=M$ in general, and therefore we find exactly and uniquely the $M$ positions as the roots of the polynomial $R(X)$.

4.1. Implementation notes. The structure of $\mathbf{A}$ can be simplified by performing the following factorization:

$$
\mathbf{A}=\mathbf{H} \boldsymbol{\mu} \mathbf{a},
$$

Copyright $\odot$ by SIAM. Unauthorized reproduction of this article is prohibited. 


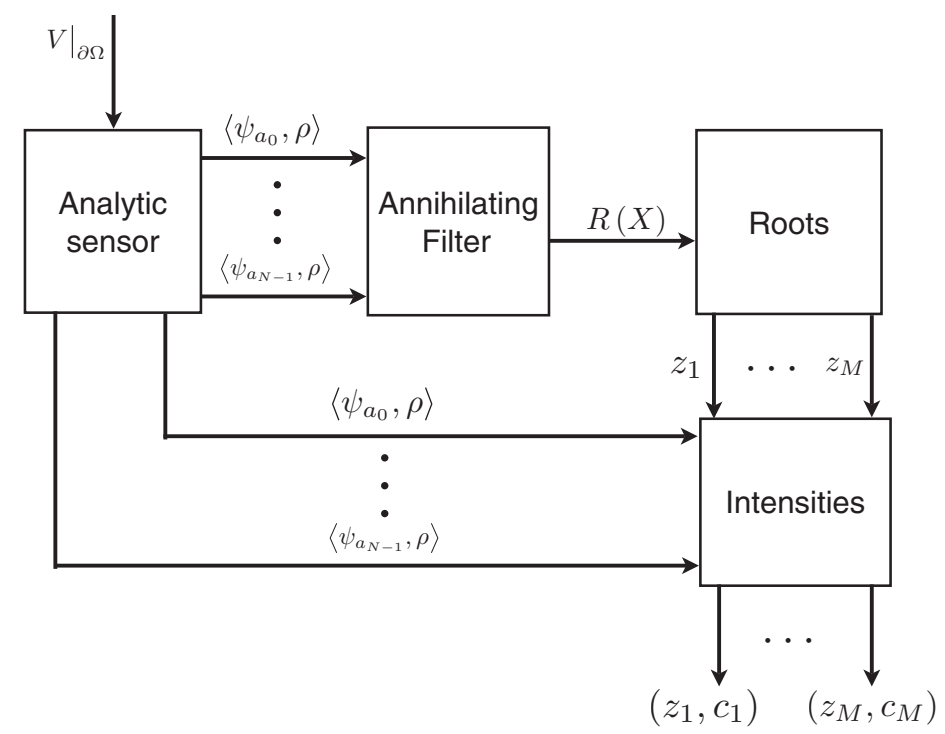

Fig. 4. A flow chart of the proposed algorithm to determine the positions of the pointwise sources and their corresponding intensities.

where $\mathbf{H}$ is an $(N-M) \times N$ Toeplitz matrix representing the discrete filter $h, \boldsymbol{\mu}$ is a diagonal $N \times N$ matrix with $\mu_{n}$, for $n \in \llbracket 0, N-1 \rrbracket$, on the diagonal, and a is an $N \times(M+$ 1) Vandermonde matrix. More explicitly, the matrices $\mathbf{H}, \boldsymbol{\mu}$, and a read as follows:

$$
\begin{gathered}
\mathbf{H}=\left[\begin{array}{llllll}
h_{M} & \cdots & h_{0} & 0 & \cdots & 0 \\
0 & h_{M} & \cdots & h_{0} & \cdots & 0 \\
\vdots & \ddots & \ddots & \cdots & \ddots & \vdots \\
0 & \cdots & 0 & h_{M} & \cdots & h_{0}
\end{array}\right], \\
\boldsymbol{\mu}=\left[\begin{array}{llll}
\mu_{0} & & 0 \\
& \ddots & \\
0 & & \mu_{N-1}
\end{array}\right],
\end{gathered}
$$

and

$$
\mathbf{a}=\left[\begin{array}{lll}
a_{0}^{0} & \cdots & a_{0}^{M} \\
\vdots & \ddots & \vdots \\
a_{N-1}^{0} & \cdots & a_{N-1}^{M}
\end{array}\right]
$$

Since $\mathbf{H}$ is a convolution matrix, it is in general not unitary, even if it usually has maximal rank, and this may be detrimental to the computation of the polynomial $R(X)$ when the generalized measurements are not known with high enough accuracy. However, we observe that if we perform a singular value decomposition of $\mathbf{H}$ according to $\mathbf{H}=\mathbf{U S H}_{0}$, where $\mathbf{U}$ is an $(N-M) \times(N-M)$ unitary matrix, $\mathbf{S}$ is an $(N-M) \times$ $(N-M)$ diagonal matrix, and $\mathbf{H}_{0}$ is an $(N-M) \times N$ matrix satisfying $\mathbf{H}_{0} \mathbf{H}_{0}^{\dagger}=\mathbf{I d}$, then

$$
\mathrm{AR}=\mathbf{H} \mu \mathrm{aR}=0 \quad \Leftrightarrow \quad \mathbf{A}_{0} \mathrm{R}=\mathbf{H}_{0} \boldsymbol{\mu a R}=0
$$


whenever $\mathbf{H}$ has maximal rank; i.e., whenever $\mathbf{S}$ is nonsingular. The right-hand side linear system of equations is actually much better conditioned and this is the one that we will consider for practical implementations.

4.2. Noise issues. The algorithm of the previous section assumes perfect data, but a practical situation has to cope with noise that inevitably corrupts measured data. Consequently, we implemented the method described above with a minor adjustment to compensate for the influence of noise.

In the presence of noise, the generalized measures are distorted in such a way that the decomposition of $\mathbf{A}_{0}$, as stated ideally in (26), becomes

$$
\mathbf{A}_{0}=\mathbf{H}_{0}(\boldsymbol{\mu}+\mathbf{b}) \mathbf{a},
$$

where $\mathbf{b}$ is a diagonal matrix representing the additive noise that we assume to have zero mean. As a consequence, on the average we have the following:

$$
\mathscr{E}\left\{\left\|\mathbf{A}_{0} \mathrm{R}\right\|^{2}\right\}=\left\|\mathbf{H}_{0} \boldsymbol{\mu a R}\right\|^{2}+\mathscr{E}\left\{\left\|\mathbf{H}_{0} \mathbf{b a R}\right\|^{2}\right\} .
$$

Given that the localization parameters are obtained by finding any nontrivial vector $\mathrm{R}$ of length $M+1$, such that $\mathbf{H}_{0} \boldsymbol{\mu} \mathrm{aR}=0$, we see from the above expression that, in order to avoid a systematic bias due to the noise, it is advisable to solve the following minimization problem:

$$
\min _{\mathrm{R} \in \mathbb{C}^{M+1}}\left\|\mathbf{A}_{0} \mathrm{R}\right\|^{2} \quad \text { subject to } \quad \mathscr{E}\left\{\left\|\mathbf{H}_{0} \mathbf{b a R}\right\|^{2}\right\}=\text { constant. }
$$

This way, we actually minimize an expression that is close to $\left\|\mathbf{H}_{0} \boldsymbol{\mu a R}\right\|^{2}$, which would be the one that is set to zero in the noiseless annihilation problem. In order to perform the optimization (28), we need to set a hypothesis on the covariance matrix of the noise. If we assume this covariance to be $\sigma^{2} \mathbf{I d}$ (white noise hypothesis), we have

$$
\begin{aligned}
\mathscr{E}\left\{\left\|\mathbf{H}_{0} \mathbf{b a R}\right\|^{2}\right\} & =\mathscr{E}\left\{\mathrm{R}^{\dagger} \mathbf{a}^{\dagger} \mathbf{b}^{\dagger} \mathbf{H}_{0}^{\dagger} \mathbf{H}_{0} \mathbf{b a R}\right\} \\
& =\sigma^{2} \mathrm{R}^{\dagger} \mathbf{a}^{\dagger} \operatorname{diag}\left\{\mathbf{H}_{0}^{\dagger} \mathbf{H}_{0}\right\} \mathbf{a R} .
\end{aligned}
$$

The variance $\sigma^{2}$ is completely determined by the signal-to-noise ratio (SNR) of the generalized measures. Of course, in the absence of noise, (28) yields the exact position parameters.

4.3. Accuracy of the retrieval. To evaluate the performance of the proposed algorithm in the presence of noise, we compute the Cramér-Rao lower bounds for the setting with the additive white Gaussian noise hypothesis [8]. Given noisy generalized measures, these bounds establish the minimal covariance matrix of any unbiased estimate of the position and intensity parameters. lowing:

The signal model describing the noisy generalized samples, $g\left(\mathbf{x} ; a_{n}\right)$, is the fol-

$$
g\left(\theta ; a_{n}\right)=f\left(\theta ; a_{n}\right)+v_{n}+i w_{n},
$$

where we have defined

$$
\begin{gathered}
f\left(\theta ; a_{n}\right)=\mu_{n} \quad \text { and } \quad \theta=[\operatorname{Re}(\mathbf{z}), \operatorname{Im}(\mathbf{z}), \operatorname{Re}(\mathbf{c}), \operatorname{Im}(\mathbf{c})]^{\mathrm{T}} \\
\text { with } \mathbf{z}=\left[z_{1}, z_{2}, \ldots, z_{M}\right] \quad \text { and } \quad \mathbf{c}=\left[c_{1}, c_{2}, \ldots, c_{M}\right] .
\end{gathered}
$$

Copyright @ by SIAM. Unauthorized reproduction of this article is prohibited. 


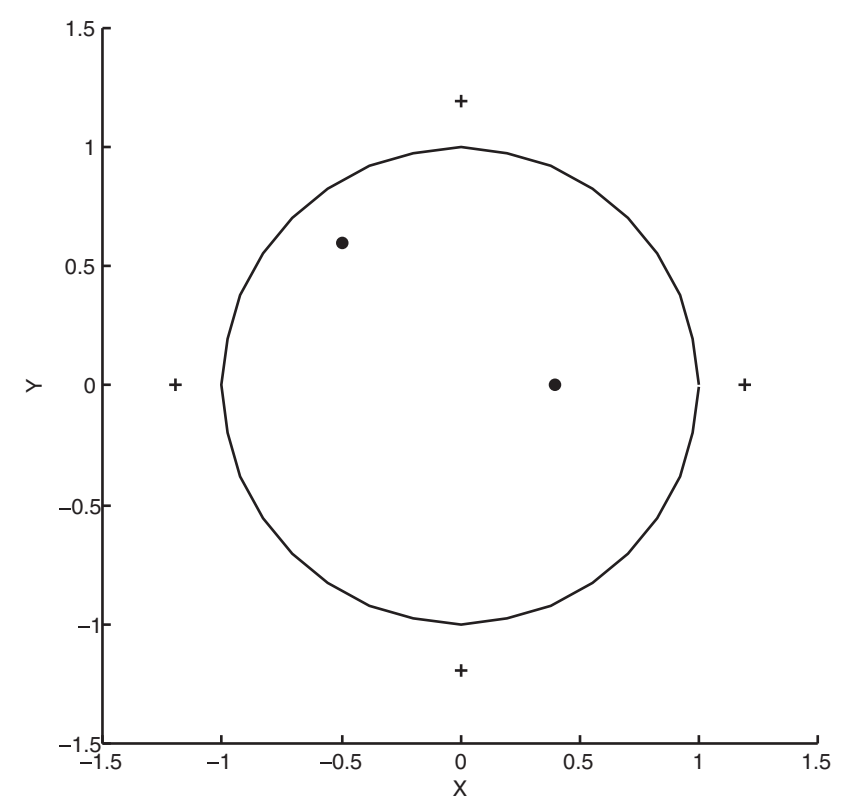

FIG. 5. The source configuration with two pointwise sources located at $\left(x_{1}, y_{1}\right)=(0.7,-0.3)$ and $\left(x_{2}, y_{2}\right)=(-0.5,0.6)$. Moreover, the singularities of the analytic sensors, located at $e^{i n \frac{\pi}{2}}$ for $n \in \llbracket 0,3 \rrbracket$, are indicated as well.

Moreover, $v_{n}$ and $w_{n}$ are independent normally distributed random variables with expected value 0 and variance $\sigma^{2}$.

In order to compute these lower bounds, we determine the Fisher information matrix, $\mathbf{J}=\left[J_{k, l}\right]_{k, l \in \llbracket 1,2 M \rrbracket}$, corresponding to (30), which reads as follows:

$$
\mathbf{J}=\frac{1}{\sigma^{2}} \sum_{n=0}^{N-1}\left[\begin{array}{c}
\nabla_{\theta} \operatorname{Re}\left(f\left(\theta ; a_{n}\right)\right) \\
\nabla_{\theta} \operatorname{Im}\left(f\left(\theta ; a_{n}\right)\right)
\end{array}\right]\left[\begin{array}{c}
\nabla_{\theta} \operatorname{Re}\left(f\left(\theta ; a_{n}\right)\right) \\
\nabla_{\theta} \operatorname{Im}\left(f\left(\theta ; a_{n}\right)\right)
\end{array}\right]^{\mathrm{T}}
$$

The Cramér-Rao bounds are the diagonal elements of $\mathbf{J}^{-1}$.

\section{Results.}

5.1. Simulation. In many application problems, the sources can be modeled using unit dipoles. As a consequence, we performed simulations using such dipoles. The localization is the most important aspect in many applications. Hence, when simulating, we consider only the estimation of the position parameters.

We compared the obtained localization errors with the theoretical lower bounds that we computed using (32) in a function of the signal-to-noise ratio (SNR $=10 \log$ $\frac{\sum_{n} \mu_{n}^{2}}{\sum_{n}\left|v_{n}+i w_{n}\right|^{2}}$. Figures 5, 6(a), and 6(b) depict the setup, sample estimations, and corresponding lower bounds.

We see that up to a certain SNR (around $15 \mathrm{~dB}$ ) the algorithm performs well; i.e., it reaches the theoretical Cramér-Rao lower bound. We can thus say that the CramérRao bounds provide a good estimation of the performance of the localization. Further increasing the amount of noise increases the bound and the experimental estimation errors. Preliminary experiments indicate that even simple denoising techniques, such as a moving average, can further improve the localization. 


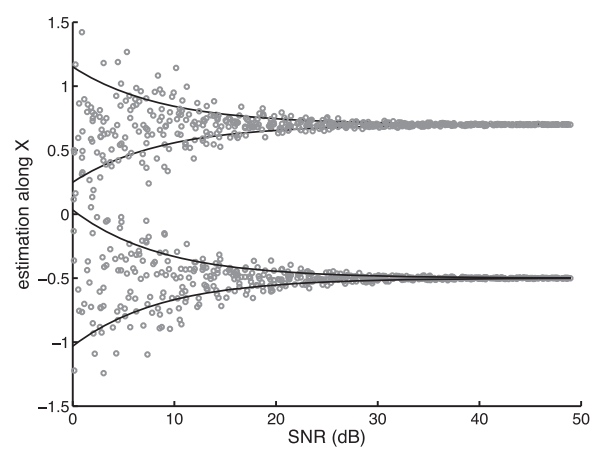

(a)

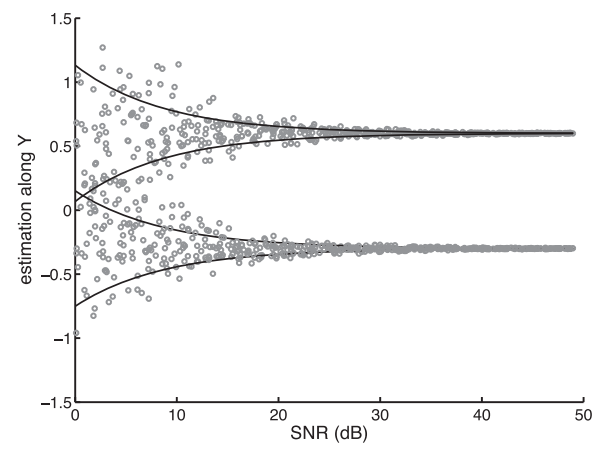

(b)

FIG. 6. 6(a) and 6(b) depict the lower bounds of any unbiased estimator (solid line) and some sample estimations (grey circles) obtained through our technique for $x_{i}$ and $y_{i}$, respectively, with $i \in\{1,2\}$. Moreover, we used the following four analytic sensors: $\psi_{a_{n}}=\frac{1}{z-1.1 e^{i \frac{\pi}{2} n}}$, with $n \in \llbracket 0,3 \rrbracket$.

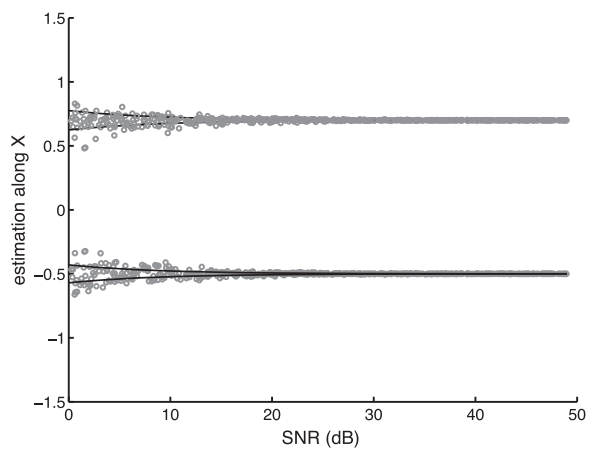

(a)

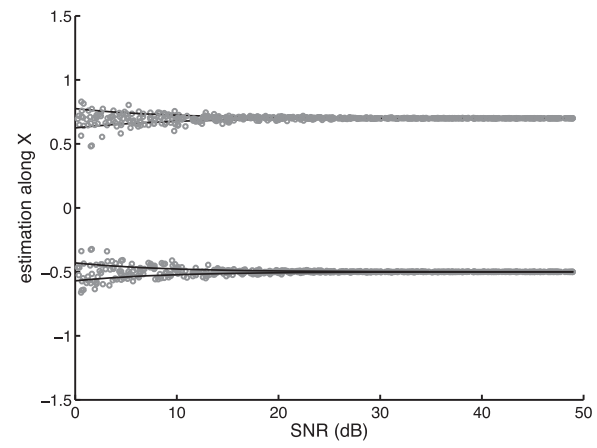

(b)

FIG. 7. 7(a) and 7(b) depict the lower bounds of any unbiased estimator (solid line) and some sample estimations (grey circles) obtained through our technique for $x_{i}$ and $y_{i}$, respectively, with $i \in \llbracket 1,2 \rrbracket$ using the following 50 analytic sensors: $\psi_{a_{n}}=\frac{1}{z-1.1 e^{i \frac{\pi}{25} n}}$, with $n \in \llbracket 0,49 \rrbracket$.

The number of analytic sensors is another parameter that influences the theoretical and experimental errors. Figures $7(\mathrm{a})$ and $7(\mathrm{~b})$ show the lower bounds for estimating $x_{i}$ and $y_{i}$ using 50 analytic sensors. Compared to Figures 6(a) and 6(b), we note that the bounds are narrower and that the sample estimations are closer to the true values of the estimated parameters for lower SNRs.

Another factor that influences the theoretical minimal and experimental errors is the position of the dipole. Figures $8(\mathrm{a})$ and $8(\mathrm{~b})$ show the sum of the Cramér-Rao bounds, plotted as a gray-scale intensity in the resulting image, when shifting a dipole through a unit-disk using a squared grid.

Figure 8(a) clearly depicts the local influence of the analytic sensors. That is, the closer the dipole is located to a pole, $a_{n}$, the smaller the localization errors are. Thus, the analytic sensors properly sense the dipole's influence when the source and sink are close to any of the poles. Hence, when the dipole approaches the center of the disk, the localization errors increase, as depicted in Figures 8(a) and 8(b). Moreover, when using more analytic sensors the estimation error decreases. This is indicated by the fact that Figure 8(b) is darker than Figure 8(a).

Copyright (C) by SIAM. Unauthorized reproduction of this article is prohibited. 


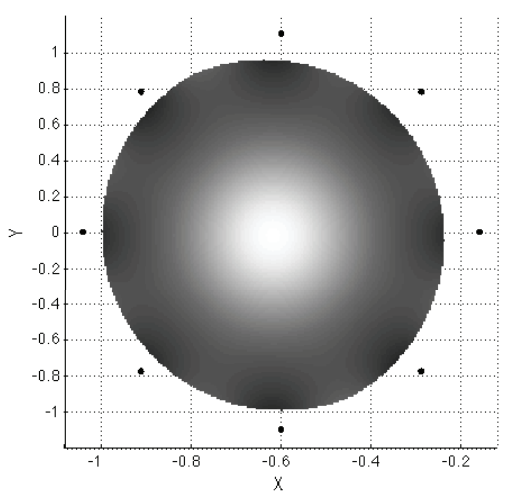

(a)

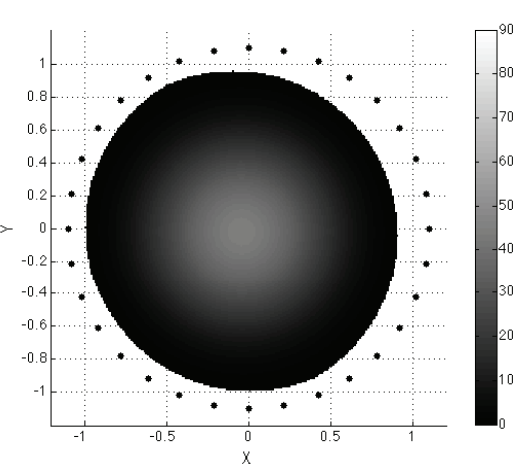

(b)

FIG. 8. 8(a) and 8(b) depict the sum of the minimal estimation errors, indicated by the grayscale intensity, when shifting a dipole through a unit-disk using a squared grid with step 0.01. The displacement of the point source with intensity -1 with respect to the point source with intensity 1 is $(-0.05,0.1)$ and the dipole's intensity is 1 . The noise is Gaussian with expected value 0 and variance 0.06. The analytic sensors are, respectively, $\psi_{a_{n}}=\left(z-1.1 e^{i n \frac{\pi}{4}}\right)^{-1}$ with $n \in \llbracket 0,7 \rrbracket$ and $\psi_{a_{n}}^{\prime}=\left(z-1.1 e^{i n \frac{\pi}{16}}\right)^{-1}$ with $n \in \llbracket 0,31 \rrbracket$.

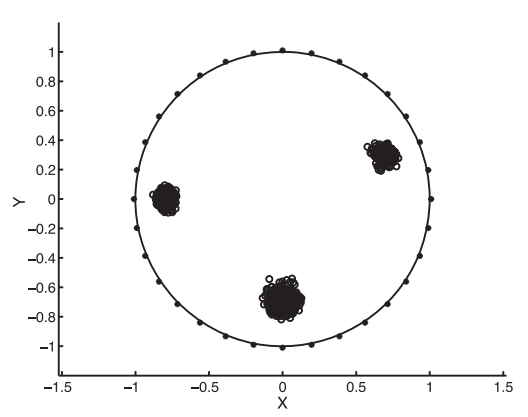

(a)

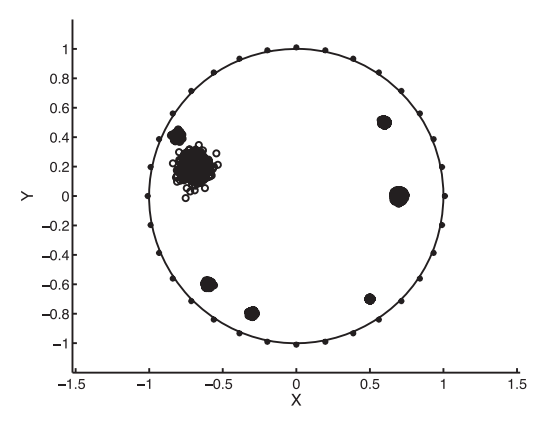

(b)

FIG. 9. 9(a) and 9(b) depict the localization of 3 and 7 point sources, respectively. The noise added to the generalized measures is Gaussian with zero mean such that the localization is performed at $15 \mathrm{~dB}$ and $40 \mathrm{~dB}$, respectively. The analytic sensors are $\psi_{a_{n}}^{\prime}=\left(z-1.01 e^{i n \frac{\pi}{16}}\right)^{-1}$ with $n \in \llbracket 0,31 \rrbracket$.

5.2. Multipoles. Until now we have located a dipole using the proposed method. However, as schematically depicted in Figure 4, we can also use the algorithm to localize multipoles, that is, $M>2$. Figures $9(\mathrm{a})$ and $9(\mathrm{~b})$ are scatterplots of the estimated positions of 3 and 8 point sources. The estimations are performed using noisy generalized measures, which are generated using (30), and 32 analytic sensors.

We clearly see that the algorithm is able to identify the "active regions." Sensitivity to noise increases with the number of sources to estimate.

6. Extension to 3D. We have shown how to efficiently localize point sources in the 2D case; we will show how we can adapt the proposed method for the $3 \mathrm{D}$ case. Basically, we can account for a z-component using two approaches: a multiplanar approach [11] and the use of a second set of test functions to reconstruct the missing z-component. Here we exploit the second approach, because we find it more consistent with our analytic sensing theory.

Copyright (C) by SIAM. Unauthorized reproduction of this article is prohibited. 
First, we note that the computation of the generalized sample as stated in Theorem 1 remains formally the same in the $3 \mathrm{D}$ case. However, the integral on the right-hand side is now a surface integral.

Second, we consider two sets of analytic sensors. The first set $\left\{\psi_{a_{n}}\right\}_{a_{n} \notin \Omega}$ contains the same sensors ${ }^{3}$ as described in (11); that is, $\psi_{a_{n}}(x, y, z)=\frac{1}{x+i y-a_{n}}$. Next, we apply the algorithm as schematized in Figure 4. This yields the projections on the XY-plane of the position parameters, $\left\{\left(x_{1}, y_{1}\right), \cdots,\left(x_{M}, y_{M}\right)\right\}$, and the corresponding intensities, $\left\{c_{1}, \ldots, c_{M}\right\}$.

The second set $\left\{\psi_{a_{n}}^{\prime}\right\}$ contains the following analytic- $-\Delta \psi^{\prime}=0$ - sensors:

$$
\begin{aligned}
\psi_{a_{n}}^{\prime}(x, y, z) & =z \psi_{a_{n}}(x, y, z) \\
& =\frac{z}{x+i y-a n} .
\end{aligned}
$$

The generalized samples, which are computed using Theorem 1 and (33), read as follows:

$$
\left\langle\psi_{a_{n}}^{\prime}, \rho\right\rangle=\sum_{m=1}^{M} c_{m} \frac{z_{m}}{x_{m}+i y_{m}-a_{n}} .
$$

Hence, these measures are linear in $z_{m}$. Since we have access to the values of $x_{m}, y_{m}$, and $c_{m}$ for $m \in \llbracket 1, M \rrbracket$, we can determine the corresponding $z$-coordinates by solving the following system of linear equations:

$$
\left\langle\psi_{a_{n}}^{\prime}, \rho\right\rangle=\sum_{m=1}^{M} c_{m} \frac{z_{m}}{x_{m}+i y_{m}-a_{n}}, \quad n \in \llbracket 0, N-1 \rrbracket .
$$

7. Conclusion. We proposed "analytic sensing" as a novel noniterative algorithmic framework for identifying parametric source models from planar Cauchy measurements. We extend the reciprocity gap concept by a particular choice of test functions (sensors) that allow us to subsequently deploy annihilation filters to solve the nonlinear problem associated with the localization problem.

We demonstrated the feasibility of the proposed algorithm by experimental simulations in a setup including noise degradation. The method reaches the theoretical best performance as predicted by the Cramér-Rao bounds for a large range of SNR. These results are reassuring as no other unbiased estimator can do better. An attractive property of our algorithm is also that it provides a direct estimation even for multipole configurations.

\section{REFERENCES}

[1] S. Andrieux And A. Ben ABda, Identification of planar cracks by complete overdetermined data: Inversion formulae, Inverse Problems, 12 (1996), pp. 553-564.

[2] L. Baratchart, A. Ben Abda, F. Ben Hassen, and J. Leblond, Recovery of pointwise sources or small inclusions in $2 D$ domains and rational approximation, Inverse Problems, 21 (2005), pp. 51-74.

[3] L. Baratchart, J. Leblond, F. Mandréa, and E. B. Saff, How can the meromorphic approximation help to solve some $2 D$ inverse problems for the Laplacian?, Inverse Problems, 15 (1999), pp. 79-90.

\footnotetext{
${ }^{3}$ The poles $a_{n}$ are chosen in such a way that the vertical line that passes through $\left(\operatorname{Re}\left(a_{n}\right), \operatorname{Im}\left(a_{n}\right), 0\right)$ does not intersect $\Omega$.
} 
[4] T. Blu, P.-L. Dragotti, M. Vetterli, P. Marziliano, and L. Coulot, Sparse sampling of signal innovations, IEEE Signal Process. Mag., 25 (2008), pp. 31-40.

[5] J. R. CANNON AND R. E. EWING, The locations and strengths of points sources, Improperly Posed Boundary Value Problems (Research Notes in Mathematics), 1 (1975), pp. 39-53.

[6] M. Chafik, A. El Badia, And T. Ha-Duong, On some inverse EEG problems, Inverse Problems Eng. Mech. II (2000), pp. 537-544.

[7] A. El Badia And T. HA-DuOng, An inverse source problem in potential analysis, Inverse Problems, 16 (2000), pp. 651-663.

[8] K. Fununaga, Introduction to Statistical Pattern Recognition, Academic Press, New York, 1972.

[9] D. B. GeSelowitz, On bioelectric potentials in an inhomogeneous volume conductor, Biophys. J., 7 (1967), pp. 1-11.

[10] D. Kandaswamy, T. Blu, L. Spinelli, C. Michel, and D. Van De Ville, Ee source localization by multi-planar analytic sensing, in Proceedings of the Fifth IEEE International Symposium on Biomedical Imaging: From Nano to Macro (ISBI'08), Vol. 6701, Paris, France, 2008, pp. 1075-1078.

[11] D. Kandaswamy, T. Blu, L. Spinelli, C. Michel, and D. Van De Ville, EeG source localization by multi-planar analytic sensing, in Proceedings of the Fifth IEEE International Symposium on Biomedical Imaging: From Nano to Macro (ISBI'08), Paris, France, 2008, pp. $1075-1078$.

[12] D. Kandaswamy, T. Blu, and D. Van De Ville, Analytic sensing: Direct recovery of point sources from planar Cauchy boundary measurements, in Proceedings of the SPIE Conference on Mathematical Imaging: Wavelet XII, Vol. 6701, San Diego, CA, 2007, pp. 67011Y$1-67011 \mathrm{Y}-6$.

[13] V. A. Kozlov, V. G. Maz'Ya, And A. V. Fomin, An iterative method for solving the Cauchy problem for elliptic equations, Comput. Math. Phys., 31 (1991), pp. 45-52.

[14] J. Kybic, M. Clerc, T. Abboud, O. Faugeras, R. Keriven, and R. Papadopoulo, A common formalism for the integral formulations of the forward EEG problem, IEEE Trans. Medical Imaging, 24 (2005), pp. 12-28.

[15] C. M. Michel, M. M. Murray, G. Lantz, S. Gonzalez, L. Spinelli, and R. Grave de Peralta, EEG source imaging, Clin. Neurophysiol., 115 (2004), pp. 2195-2222.

[16] K. Miller, Stabilized numerical prolongation with poles, SIAM J. Appl. Math., 18 (1970), pp. 346-363.

[17] M. Renardy and R. C. Rogers, An Introduction to Partial Differential Equations, Springer, New York, 2004.

[18] L. Spineldi, S. G. Andino, G. Lantz, M. Seeck, and C. M. Michel, Electromagnetic inverse solutions in anatomically constrained spherical head models, Brain Topography, 6 (2000), pp. $115-125$.

[19] A. N. Tikhonov, On the stability of inverse problems, Dokl. Akad. Nauk SSSR, 39 (1943), pp. 195-198.

[20] S. Vessela, Locations and strengths of point sources: Stability estimates, Inverse Problems, 8 (1992), pp. 911-917.

[21] M. Vetterli, P. Marzilliano, And T. Blu, Sampling signals with finite rate of innovation, IEEE Trans. Signal Process., 50 (2002). 